\title{
AN UNUSUAL ORBITOCRANIAL THROUGH AND THROUGH PENETRATING INJURY BY IN SITU TETA: CAN WE DO BETTER FOR THIS TYPE OF UNFORTUNATE PATIENT?
}

\author{
CHOWDHURY FH ${ }^{1}$ HAQUE MR ${ }^{2}$, ISLAM MS ${ }^{3}$, KAWSAR KA ${ }^{4}$, ALAM MK $^{5}$
}

\begin{abstract}
:
Through and through orbitocranial penetrating injury by in situ teta is extremely rare. We managed such a case in Dhaka Medical college hospital with many limitations. In spite of all possible supports that were available in our set up, patient did not survive. Presentation, investigations, surgical and other supportive management will be highlighted in this case report.
\end{abstract}

Key Words: Orbitocranial injury, penetrating injury, teta.

J Dhaka Med Coll. 2009; 18(2) : 185-187

\section{Introduction:}

Penetrating orbitocranial injuries are rare. They may appear to be associated with intracranial injuries with or without secondary complications, may ultimately prove fatal.

We report a case of very unusual (probably the first reported case) orbitocranial through and through penetrating injury by teta which was in situ before operation.

\section{Case Report:}

A fifteen years old young boy presented with the $\mathrm{H} / \mathrm{O}$ penetrating injury in his right eye by a long teta inflicted by robbers during robbing him at local market. Initially he was managed in local hospital. Ten hours later, he presented to us. During this period he developed generalized convulsion for four times. On examination, we found teta penetrating his right eye with handle and small part of metallic portion remaining outside (figure-1). On occipital region or other parts of scalp, there was no exit wound but apex of teta could be palpated under the skin in right occipital region. There was no other external injury. His vital signs were stable, GCS was 08(E1M5V2). There was no obvious limb weakness on painful stimulus.
We advised urgent X-ray skull, CT scan of head with bony window and Cerebral angiography. The patient party could not afford cerebral angiography on emergency basis. X-ray (figure2) and CT scan showed that teta has passed under the right orbital plate through the eye ball, superior orbital fissure, right temporal, occipital lobe and occipital bone above and right to midline. There was no intracranial haematoma. Reverse spike of teta was clearly

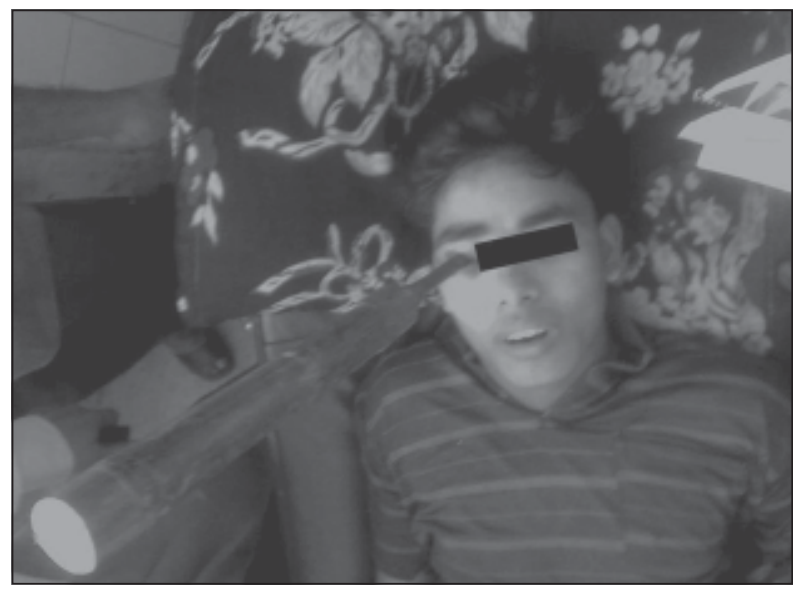

Fig.-1: Preoperative picture of patient with teta passing through the orbit.

1. Resident, Department of Neurosurgery, Dhaka Medical College Hospital, Dhaka.

2. Associate Professor, Department of Neurosurgery, Dhaka Medical College Hospital, Dhaka.

3. Resident, Department of Neurosurgery, Dhaka Medical College Hospital, Dhaka.

4. Resident, Department of Neurosurgery, Dhaka Medical College Hospital, Dhaka.

5. Assistant Professor, Department of Orthopaedics, Dhaka Medical College, Dhaka.

Correspondence to: Dr. Chowdhury Forhad Hossain. 


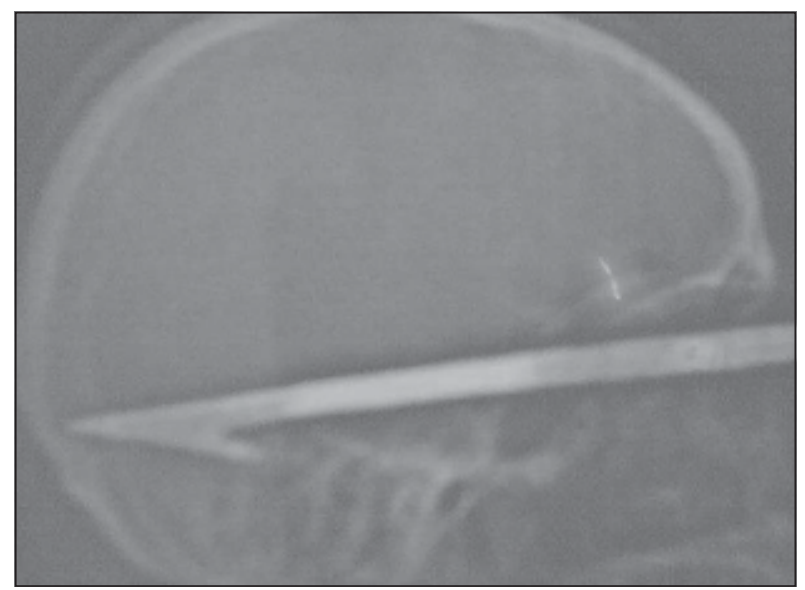

Fig.-2: Preoperative X-ray skull lateral view showing teta passing through orbit and cranial cavity.

visible. We also consulted with ophthalmologists and they participated in the surgery.

Patient underwent emergency operation under GA. Part of teta exterior to eye right eye was cut with metal cutter. A right sided pterional craniotomy was done and middle cerebral and other related vessels were found intact and away from the teta. Then through a right occipital craniotomy teta was removed carefully, followed by removal of dead brain and tissue with toileting as far as possible.

Postoperatively, anesthesiologist suggested for ICU support but unfortunately ICU bed was filled up; patient was managed in post operative room. Postoperatively, convulsion was going on and it was managed by i.v. fosphenytoin. GCS remained unchanged. On second $P O D$, the patient developed fever and convulsion, respiratory distress, GCS deteriorated to $\mathrm{E} 1 \mathrm{M} 4 \mathrm{~V} 1=06$. Later on, patient could be shifted to ICU. In spite of all troublesome efforts in our set up, patient expired on fourth POD.

\section{Discussion:}

In this case teta has passed through the superior orbital fissure, which is one of the natural pathways for orbitocranial penetrating foreign body. For proper management of such a patient complete neurological examination with x-ray of skull is needed. ${ }^{1}$ A CT scan is also needed to delineate the trajectory, position, relation with anatomical structures with the foreign body. ${ }^{2}$ It is also helpful to see any haematoma or other pathology that may develop with the penetrating injury. Such type of injury may also be associated with cerebral arterial or venous sinus injury. ${ }^{3,5,6}$ So in our case cerebral angiography was advised but the patient's party could not afford it. So, during operation vascular injuries were excluded by pterional and occipital craniotomy before removal of teta.

The significant reasons for surgery in such a case are

a) To remove intracranial haematoma,

b) To remove necrotic brain to prevent infection, mass affect and ischaemia, ${ }^{4,8}$

c) To control active bleeding,

d) To remove foreign body to prevent infection and its further complications. ${ }^{8}$

Approach to surgery varies in such a case; some are conservative while others are more aggressive. ${ }^{4,7}$ Before operation, we discussed two options regarding management of the patient. One was for emergency operation and the other was for initial conservative management followed by surgery few weeks later.

Points in favor of emergency removal of teta were-

1. In such a case, surgical principle is in favor of removal of foreign body followed by surgical toileting.

2. Conservative management in such a case will invite infection, septicemia, abscess formation etc.

3. It would be very odd looking and nuisance to keep teta in situ during conservative management period.

4. Pressure from the patient's party and other groups (newsmen) for removal of teta were also considered.

The second opinion was in favour of initial conservative management with aggressive antibiotic, anticonvulsant and other supportive therapy, then removal of teta on a later date after formation of wall (gliosis \& 
fibrosis) around it with adopted brain. Second group put their defense on the point of death from infection that patient might die from infection during conservative treatment period but one should not forget that emergency can kill the patient and has got more chance to iatrogenic neurological deterioration. They also argued that human body has wonderful adaptation capacity even with this type of teta in brain and chance of late infection is less. ${ }^{9}$ Finally, the decision was taken in favor of emergency surgery but ultimately patient did not survive.

\section{Conclusion:}

This type of orbitocranial injury is probably extremely rare. There is no trail based management guideline for such a patient. Management given to this patient was probably inadequate for the survival of the young guy. Is it possible to set up a better management guideline as well as improvement of our set up, for such an unfortunate patient?

\section{Reference:}

1. Devi B Indira, Bhatia Sanjay, Kak Vijay K: Penetrating orbitocranial injuries-Report of two cases. Indian journal of Ophthalmology 1993;41:84-6.

2. De Villiers JC and Grant AR. Stab wound of craniocervical junction. Neurosurgery 1985;71:930-936

3. De Villiers JC and Grant AR. Stab wound of the brain and skull. In: Viken PJ, Bruyn GW,eds. Hand book of clinical neurology, vol 23, Newyork, NY: Elsevier Science Publishing; 1975:407-503.

4. Federico C Vinas, Julie Pilitsis: Penetrating Head Trauma. emedicine June 20, 2006.

5. Hadded FS, Haddded GF and Taha J. Traumatic intracranial aneurysm by missiles: Their presentation and management. Neurosurgery 1991; 28:1-7.

6. Kieck CF, De Villiers JC. Vascular lesion due to transcranial stab wounds 1984;60:42-46.

7. Kaufman HH, Schwab K, Salazar AM: a national survey of neurosurgical care for penetrating injury. Surg Neurol 1991 Nov;36(5):370-7

8. Maruya J, Yamamoto K, Kaniko U: Brain Abscess following transorbital penetrating injury due to bamboo fragments. Case report: Neurol Med Chir2002;42:143-146.

9. Misra M, Rath S, Acharya B, Mohanty SC. An unusual case of orbitocranial gunshot wound. Indian J Opthalmol 1985;33:105-7 